\title{
LncRNA-ANCR regulates the cell growth of (a) CrossMark osteosarcoma by interacting with EZH2 and affecting the expression of p21 and p27
}

Fei Zhang and Hao Peng*

\begin{abstract}
Background: Osteosarcoma (OS) is one of the most common malignant tumors developed in the bone. EZH2 has been found to play pivotal roles in the development of various cancers. LncRNA-ANCR (anti-differentiation ncRNA) has been reported to interact with $\mathrm{EZH} 2$ and regulated osteoblast differentiation. Our study aimed to investigate the effect of InCRNA-ANCR on the tumorigenesis of osteosarcoma and explore the underlying molecular mechanism.

Methods: RT-PCR was performed to detect the messenger RNA (mRNA) levels of IncRNA-ANCR, EZH2, p21, and p27 in OS tissues and cell lines. The cell proliferation, transwell invasion, and migration assays were conducted to evaluate the influence of IncRNA-ANCR depletion on the growth of OS cells. RNA pull-down assay was carried out to detect the interaction between IncRNA-ANCR and EZH2. Correlation between the expression of IncRNA-ANCR and the expression of EZH2 were analyzed by cross-tabulation.

Results: LnCRNA-ANCR is highly expressed in both OS tissues and cell lines. Reduced expression of IncRNA-ANCR inhibited the cell proliferation, invasion, and migration of OS cells. The cell apoptosis rate was also increased with the overexpression of IncRNA-ANCR. Mechanistically, downregulation of IncRNA-ANCR reduced the mRNA level of EZH2 and increased the expression of p21 and p27 at both mRNA and protein levels. LnCRNA-ANCR interacted with EZH2 and their expression abundance was positively correlated in OS patients.
\end{abstract}

Conclusion: LnCRNA-ANCR inhibited the cell proliferation, migration, and invasion of OS cells possibly through interacting with $\mathrm{EZH} 2$ and regulating the expression of p21 and p27.

Keywords: LncRNA-ANCR, Osteosarcoma, EZH2, p21, p27

\section{Background}

Osteosarcoma, which is also called osteogenic sarcoma (OGS), is one of the most common malignant tumors developed in bone. OS accounts for about $60 \%$ cases of all the bone cancerous tumors in both adolescence and childhood [1-3]. The patients with OS usually have a high incidence of lung metastasis, and the 5-year survival rate is less than $30 \%$ for patients suffering OS combined with lung metastasis [4-6]. Therefore, identifying the molecular targets involved in the development of OS and developing treatment strategies is quite necessary.

\footnotetext{
* Correspondence: owujnl@sina.com

Department of Orthopaedics Surgery, Renmin Hospital of Wuhan University, Wuhan 430060, Hubei, China
}

Long non-coding RNAs (lncRNA) are a group of nonprotein coding RNAs with a length longer than 200 nucleotides [7], which is different from that of short interfering RNAs (siRNAs), microRNAs (miRNAs), Piwi-interacting RNAs (piRNAs), small nucleolar RNAs (snoRNAs), and other short RNAs. Previous study has shown that the expression levels of many lncRNAs were altered in OS tissue [8]. A recent study reported that downregulation of IncRNA TUG1 reduced the proliferation rate and increased apoptosis rate in OS cell [9], which indicated that lncRNAs may be considered as biomarkers for the diagnosis of OS and potential molecular targets for the treatment.

Enhancer of Zeste Homolog 2 (EZH2), a histone methyltransferase, has been found to play pivotal roles in the development of various cancers $[10,11]$. EZH2 is highly 
expressed in solid tumors to initiate cell proliferation [12], and the high expression of EZH2 is negatively correlated with the patients' outcome [13, 14]. It has been reported that overexpression of EZH2 increased the trimethylation of H3K27 on the promoters of p21, which facilitated the p53 binding on the promoter and activated the expression of p21 [15]. Additionally, p27 was also negatively regulated by EZH2 [16, 17]. These findings provided important cues to understand the molecular mechanism of EZH2 in cancer development.

LncRNA-ANCR (anti-differentiation ncRNA) has been demonstrated to be involved in regulating osteoblast differentiation [18], however, the function of IncRNAANCR in osteosarcoma remains largely unknown. Additionally, it has been documented that IncRNA-ANCR interacted with EZH2 to regulate the differentiation of osteoblast. In view of the function correlation of EZH2 and IncRNA-ANCR, we hypothesized that IncRNAANCR may play critical roles in OS.

In this study, to explore the function of IncRNAANCR in OS, the expression level of lncRNA-ANCR in both OS tissues and cell lines were determined. High expression abundance of lncRNA-ANCR in OS was observed. Downregulation of lncRNA-ANCR inhibited the cell proliferation of OS cells. Further investigation found that depletion of IncRNA-ANCR suppressed the expression of EZH2 and activated p21 and p27. Positive correlation between the expression of lncRNA-ANCR and $\mathrm{EZH} 2$ was also observed in OS patients.

\section{Methods}

\section{Clinical patients and OS tissues}

The tumor specimens were obtained from 20 patients with OS who underwent resection between July 2015 and March 2016 during their hospitalization in Wu Han University affiliated hospital. The tissue samples of OS patients were graded and staged by experienced pathologists after tissues were collected. According to the medical records, 14 patients have been diagnosed with cancer metastasis among the 20 OS patients. At the same time, 20 patients with lumbar discectomy underwent resection were also selected as the control group. Ethical approval was obtained by Wu Han University affiliated hospital. We had all the necessary consent from any patients involved in the study, including consent to participate in the study where appropriate.

\section{RT-PCR}

Total RNA extraction was performed with RMeasy MiNi Kit (Qiagen, 74104, Germany). TransScript-Uni OneStep gDNA Removal and cDNA Synthesis SuperMix (ABSCI, AB452, USA) were used for reverse transcription. The RT-PCR was carried out with ABI 7500 Realtime PCR instrument and TaqMan Multiplex Master
Mix (Life Technologies, 4486295, USA). GAPDH was used as an endogenous control. The following primers were used: IncRNA-ANCR-forward: 5'-GACATTTCCT GAGTCGTCTTCGAACGGAC and reverse: 5' - TAGT GCGATTTAGAGCTGTACAAGTTTC; p21-forward: 5' - TCTGGGGTVTVACTTCTTGG and reverse: 5' -AT GTGAGGAAGGCTCAGTGG; p27-forward: 5'-GATG GGGTTCACCGTGTTAG and reverse: 5 '-CCCTTTCC AAACATCCATTG; EZH2-forward: 5' -TTGTTGGCGG AAGCGTGTAAAATC and reverse: 5 -TCCCTAGTCC CGCGCAATGAGC; GAPDH-forward: 5'-CGAGCCAC ATCGCTCAGACA and reverse: 5' -GTGGTGAAGACG CCAGTGGA. Relative RNA level was calculated using $2^{\Delta \Delta C t}$ method.

\section{Cell culture}

Human OS cell lines and osteoblast cell line hFOB1.19 were purchased from American Type Culture Collection (Rockville, MD, USA) were cultured in DMEM medium containing $10 \% \mathrm{FBS}$ at $37{ }^{\circ} \mathrm{C}$ in a humidified atmosphere with $5 \% \mathrm{CO}_{2}$.

\section{Transfection}

MG-63 and UMR-106 cells were seeded into the 6-well plates with $1 \times 10^{5}$ cells per well. Cells were cultured in DMEM medium containing 10\% FBS until $70 \%$ confluence was observed. Transfection was performed with Lipofectamine 3000 (Invitrogen, L3000015, USA) according to the manufacturer's instruction. Cells were collected after transfection for $72 \mathrm{~h}$ and were subjected to the following experiments. Lnc-ANCR siRNA design and preparation was performed by GenePharma Co. Ltd (Shanghai, China).

\section{Cell proliferation assay}

Cells were seeded in 96-well plates with $3 \times 10^{3}$ cells per well. Cell Counting Kit-8 (CCK-8) was used to detect the viability of cells according to the manufacturer's instructions. The abundance at $450 \mathrm{~nm}$ was measured. The experiment was performed with three replicates.

\section{Western blot}

Cells were lysed with the RIPA buffer and then centrifuged at $15,000 \mathrm{~g}$ for $15 \mathrm{~min}$ at $4{ }^{\circ} \mathrm{C}$. The protein concentration was measured using BCA protein assay kit (Sigma, pierce23225KIT, USA). Antibodies for EZH2 (\#4902), p27 (\#2552), p21 (\#2947), and GAPDH (\#2118) were purchased from Cell Signaling Technology.

Fluorescence-activated cell sorting (FACS) for apoptosis $3 \times 10^{5}$ of OS cells with the indicated treatment were resuspended to make the single cell suspension. Cells were stained with Fluorescein isothiocyanate-conjugated Annexin V and 7-AAD (4ABio, FXP027-100, China). 
Single staining of FITC and 7-AAD was used to set the parameters and the gate. The cell apoptosis rate was detected by the flow cytometer CytoFLEX (Beckman Coulter, Inc., Brea, CA, USA).

\section{Cell migration and invasion assay}

OS cells transfected with the indicated plasmids were collected and suspended in serum-free medium. After that, cells were added to the upper chamber covered with matrix, the lower chamber was filled with medium containing $10 \%$ FBS. After incubation at $37^{\circ} \mathrm{C}$ for $24 \mathrm{~h}$, cells below the membrane were fixed and stained. The numbers of migrated and invaded cells were counted under a microscope. The procedure of cell migration assay is the same to that of the invasion assay. The only difference is that common transwell chambers were used instead of matrix-coated ones.

\section{RNA pull-down assay}

In vitro transcription of IncRNA-ANCR was performed using T7 RNA polymerase (Ambio Life). The transcription product was purified using RNeasy Plus Mini Kit (Qiagen) treatment with DNase I (Qiagen). The purified lncRNA-ANCR was then labeled with biotin using biotin RNA Labeling Mix (Ambio Life). MG-63 and UMR-106 cells were harvested and lysed with the RIPA lysis buffer. $50 \mu \mathrm{l}$ of the lysates were aliquoted as the input, and the remaining supernatant was incubated with biotin-labeled lncRNA-ANCR at $4{ }^{\circ} \mathrm{C}$ for $2 \mathrm{~h}$. Afterwards, the M-280 Streptavidin beads (Invitrogen, CA, USA) was added into the supernatant. The mixture was incubated at $4{ }^{\circ} \mathrm{C}$ for $2 \mathrm{~h}$. At the same time, beads incubated directly with the supernatant of OS cells in the absence of biotinlabeled IncRNA-ANCR were used as the negative control. Western blot was performed to detect the binding between lncRNA-ANCR and EZH2. The level of lncRNA-ANCR was examined by PCR analysis.

\section{Statistical analyses}

Data was analyzed with SPSS 19.0 software. Enumeration data were expressed as rate or percentage. Chisquare test was used for comparisons between two groups, and one-way ANOVA was used for comparisons among multiple groups. Correlations between the expression of lncRNA-ANCR and the expression of EZH2 were analyzed by cross-tabulation. $P<0.05$ was considered to be statistically significant.

\section{Results}

LncRNA-ANCR was highly expressed in OS tissues and cells

RT-PCR was used to detect the expression level of IncRNA-ANCR in OS tissues and tumor-free tissues. Significant high expression level of lncRNA-ANCR was found in the OS tissues than that of the tumor-free tissues (Fig. 1a). In addition, the expression level of lncRNA-ANCR was also detected in a variety of human OS cells lines including MG-63, SW1353, U2OS, Saos2, 143B, Hos, MC3T3-E1, and UMR-106. As shown in Fig.1b, lncRNA-ANCR was highly expressed in OS cell lines in comparison with that of the osteoblast cell line hFOB1.19. These results suggested that IncRNA-ANCR was overexpressed in human OS. MG-63 and UMR-106 cell lines, which showed relatively higher expression levels of lncRNA-ANCR were selected for the following experiments.

\section{Downregulation of IncRNA-ANCR inhibited the cell growth of OS cells}

To determine the role of IncRNA-ANCR on the tumorigenesis of OS cells, endogenous expression of
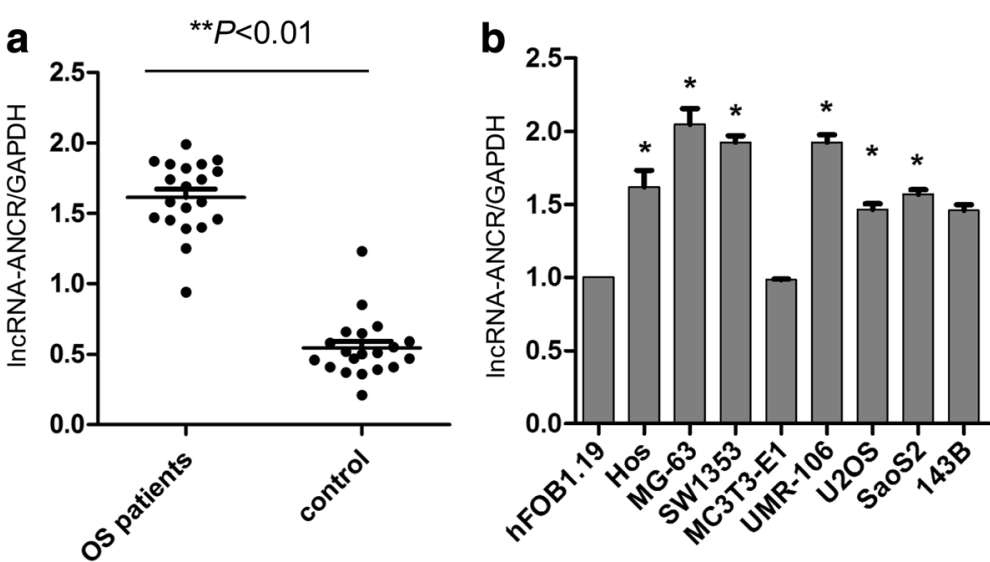

Fig. 1 LnCRNA-ANCR is highly expressed in human OS tissues and cell lines. a RT-PCT assay was performed to detect the expression of IncRNAANCR in OS patients and tumor-free patients. ${ }^{*} P<0.01$. b The expression abundance in OS cell lines and osteoblast cell line hFOB1.19 were detected. ${ }^{*} P<0.05$ 
lncRNA-ANCR was downregulated by transfecting lncRNA-ANCR-siRNA into OS cells. As shown in Fig. 2a, b, depletion of lncRNA-ANCR significantly inhibited the cell proliferation rate of both MG-63 and UMR-106 cells compared with that of the control cells. In addition, the transwell invasion and migration ability of OS cells harboring downregulated IncRNA-ANCR was also obviously decreased (Fig. 2c, d). Consistent with the inhibitory effect of IncRNA-ANCR depletion on OS cells, both MG-63 and UMR-106 cells expressing IncRNA-ANCR siRNA presented a significantly increased cell apoptosis rate (Fig. 2e). The results suggested that downregulation of lncRNA-ANCR negatively regulates the growth of OS cells.

Downregulation of IncRNA-ANCR inhibited the expression of EZH2 and activated p21 and p27

The inhibitory effect of lncRNA-ANCR downregulation on the growth of OS cells promoted us to determine the underlying molecular mechanism. It has been reported that lncRNA-ANCR interacted with EZH2 and regulated osteoblast differentiation [18]. To detect the function of IncRNA-ANCR in OS was associated with the EZH2, the endogenous expression of IncRNA-ANCR was depleted (Fig. 3a). We firstly examined the messenger RNA (mRNA) level of EZH2 in MG-63 and UMR-106 cells harboring downregulated IncRNA-ANCR. The result showed that the mRNA abundance of EZH2 was significantly decreased in OS cells with the depletion of lncRNA-ANCR (Fig. 3b). Previous studies have reported that overexpression of EZH2 promoted cell proliferation via downregulation of tumor suppressors p21 and p27 $[19,20]$. To determine the down-stream effect of the decreased EZH2 by lncRNA-ANCR knockdown, the mRNA level of both p21 and p27 was detected. As shown in Fig. 3c, d, the mRNA abundance of p21 and p27 was significantly increased in MG-63 and UMR-106 cells with the depletion of lncRNA-ANCR. Western blot analysis also showed enhanced expression level of p21 and p27 in MG-63 cells with the downregulation of lncRNA-ANCR (Fig. 3e). Similar result was also obtained in UMR-106 cells (data not shown). These data suggested that downregulation of lncRNA-ANCR inhibited the expression of EZH2 and activated the expression of p21 and p27.

LncRNA-ANCR interacted with EZH2 and the expression of IncRNA-ANCR is positively correlated with the abundance of $\mathrm{EZH} 2$ in OS

To further understand the molecular mechanism by which lncRNA-ANCR regulates the expression of EZH2, RNA pull-down assay was performed to detect the interaction between lncRNA-ANCR with EZH2. As shown in Fig.4a, biotin-labeled lncRNA-ANCR was incubated with

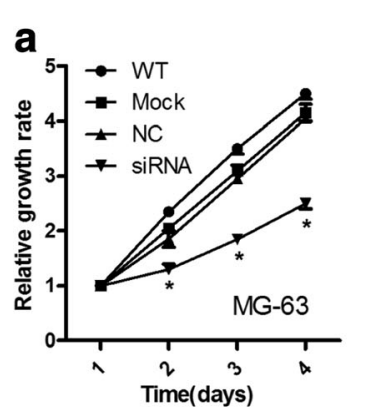

d

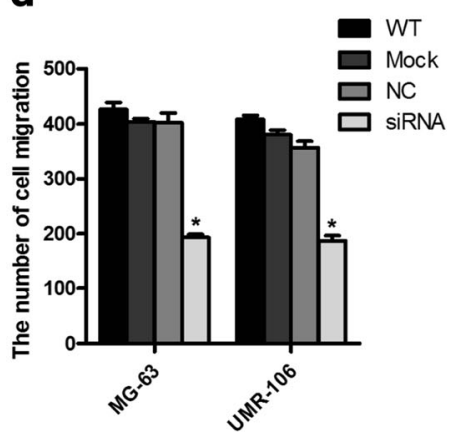

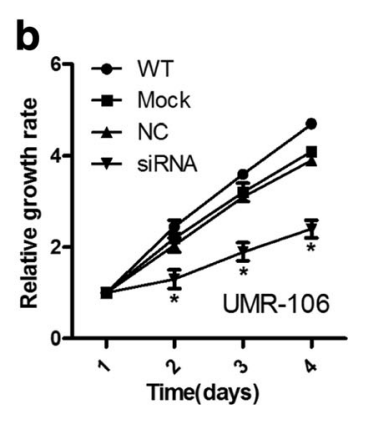

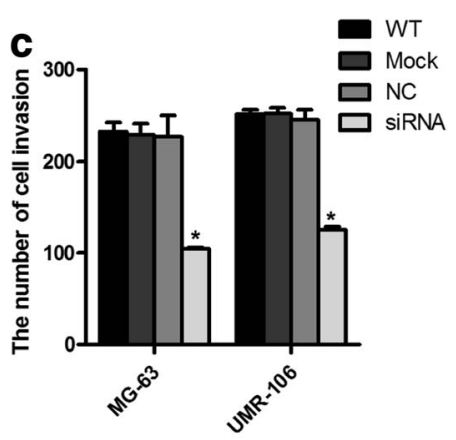

e

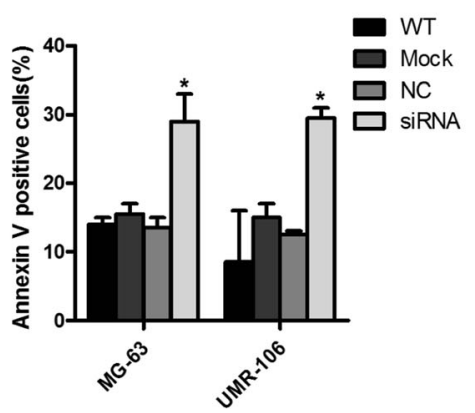

Fig. 2 Downregulation of InCRNA-ANCR inhibited the growth of OS cells. a, b The proliferation of MG-63 (a) and UMR-106 cells (b) harboring depleted InCRNA-ANCR or control vector was detected by CCK-8 assay. ${ }^{*} P<0.05$. c The data of invasion assay of MG-63 and UMR-106 cells with different treatments. ${ }^{*} P<0.05$. d The migration ability of both MG-63 and UMR-106 cells with downregulation of IncRNA-ANCR or control vector was examined. ${ }^{*} P<0.05$. e The apoptosis rate of MG-63 and UMR-106 cells with indicated treatments. ${ }^{*} P<0.05$ 

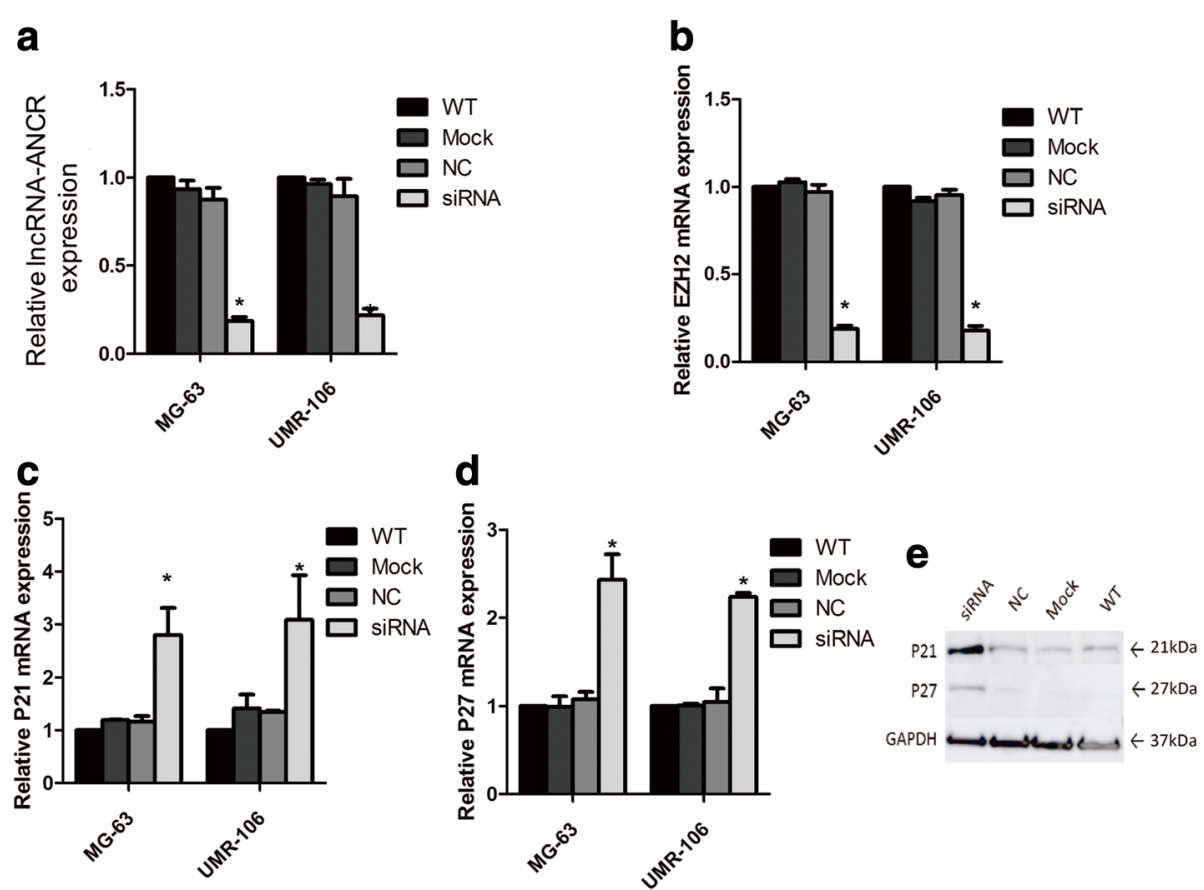

Fig. 3 Downregulation of IncRNA-ANCR suppressed the expression of EZH2 and activated p21 and p27. a The downregulation of IncRNA-ANCR by siRNA interference was validated by RT-PCR. ${ }^{*} P<0.05$. $\mathbf{b}$ The relative mRNA level of EZH2 in cells with the indicated treatments was measured by RT-PCR. ${ }^{*} P<0.05$. c, d The mRNA level of p21 (c) and p27 (d) in MG-63 and UMR-106 cells expressing IncRNA-ANCR or control vector was detected. ${ }^{*} P<0.05$. e The protein abundance of p21 and p27 in OS cells with or without IncRNA-ANCR depletion

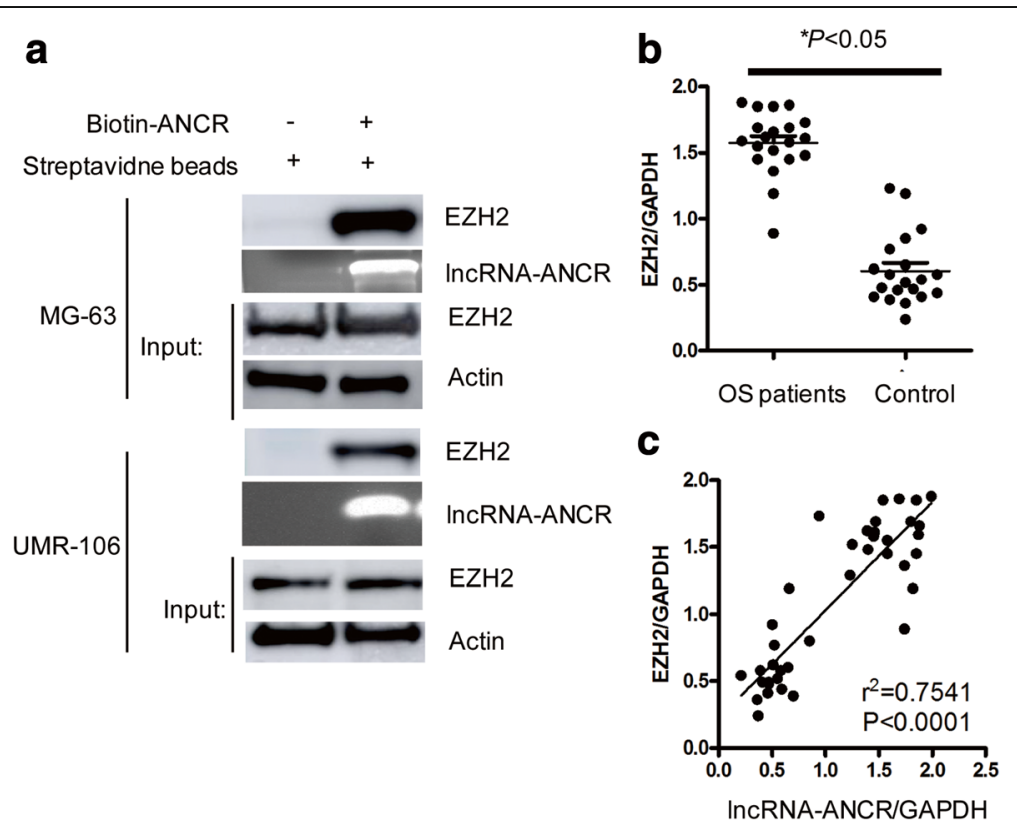

Fig. 4 LnCRNA-ANCR interacted with EZH2 and the expression of IncRNA-ANCR is positively correlated with that of EZH2. a RNA pull-down assay to show the interaction between IncRNA-ANCR and EZH2. $\mathbf{b}$ EZH2 is highly expressed in OS patients. ${ }^{*} P<0.05$. $\mathbf{c}$ The expression of IncRNA-ANCR was positively correlated with that of EZH2 
the supernatant of OS cell. The binding between lncRNA-ANCR with endogenous EZH2 was detected by western blot with anti-EZH2 antibody. The result showed that lncRNA-ANCR interacted with EZH2 (Fig 4a). We hypothesized that the interaction between lncRNA-ANCR with EZH2 may trigger the regulation of lncRNA-ANCR to EZH2.

To further confirm the correlation between IncRNAANCR and EZH2, the expression level of EZH2 in OS patients was measured. As presented in Fig. 4b, mRNA level of EZH2 was highly expressed in OS patients. The correlation analysis between lncRNA-ANCR and EZH2 showed that the expression of IncRNA-ANCR was positively correlated with that of EZH2 (Fig. $4 \mathrm{c}, r^{2}=0.7541$, $P<0.0001)$.

\section{Discussion}

As the most common primary bone tumor in both children and adolescents, OS is a devastating disease without accurate early diagnosis and efficient treatment method, which in turn leads to the low long-term survival rate [21]. The increasing morbidity of OS has been observed during the past several decades [22]. Genetic regulation plays pivotal roles in the occurrence and development of cancer. Therefore, identifying the molecular targets involved in the occurrence and development OS will benefit the diagnosis and treatment of OS. Increasing evidence has demonstrated the involvement of IncRNA in the initiation and development of OS [8]. In our study, we found that lncRNA-ANCR was highly expressed in OS tissues and cell lines. Downregulation of IncRNA-ANCR enhanced the cell apoptosis and inhibited the proliferation, migration, and invasion of OS cells. These data indicated the oncogenetic potential of IncRNA-ANCR in OS.

Previous studies have showed that EZH2 was overexpressed in a variety of human cancers, which enhanced tumorigenesis through various signaling pathways [23]. In our study, downregulation of lncRNAANCR decreased the expression abundance of EZH2, suggesting that IncRNA-ANCR positively regulates the expression of EZH2. It has been reported that EZH2 promotes the cancer cell proliferation via suppressing the expression level of the cell cycle protein including p21 and p27 [19, 20]. Consistent with these finding, we found that downregulation of lncRNA-ANCR significantly increased the mRNA and protein expression of both $\mathrm{p} 21$ and p27. These results suggested that depletion of IncRNA-ANCR negatively regulated the EZH2-p21/ p27 signaling pathway. Previous studies have shown that LncRNA-ANCR interacted with EZH2, which is important for osteoblast differentiation. Consistently, our data demonstrated the binding of lncRNA-ANCR with EZH2 in OS cells. It has been reported that overexpression of
EZH2 promoted cancer cell proliferation via downregulation of tumor suppressors p21 and p27 [19, 20]. In this study, IncRNA-ANCR interacted with EZH2 and depletion of IncRNA-ANCR activated the expression of p21 and $\mathrm{p} 27$, we hypothesized that downregulation of lncRNA-ANCR may block the recruitment of EZH2 to the promoters of $\mathrm{p} 21$ and $\mathrm{p} 27$, which attenuates the negative regulation of EZH2 on p21 and p27. This hypothesis needs further validation.

Consistent with the high expression of lncRNA-ANCR in OS patients, overexpressed EZH2 was also found in OS patients. Correlation analyses have shown that the expression of IncRNA-ANCR is positively correlated with that of EZH2. Further investigation is required to explore the upstream regulator that controls the high expression of lncRNA-ANCR and EZH2 in OS. The overexpression of lncRNA-ANCR may be a promising target for the diagnosis and treatment in OS.

\section{Conclusions}

LncRNA-ANCR was highly expressed in OS tissues and cells. LncRNA-ANCR depletion inhibited the proliferation, invasion, and migration of OS cells. Downregulation of IncRNA-ANCR decreased the abundance of EZH2 and activated the expression of both p21 and p27. The interaction between IncRNA-ANCR with EZH2 indicated that IncRNA-ANCR might exert its function via binding to EZH2. High expression of lncRNA-ANCR suggested its clinical significance in OS.

\section{Abbreviations \\ CCK-8: Cell counting kit-8; DMEM: Dulbecco's modified Eagle's medium; FACS: Fluorescence-activated cell sorting; FBS: Fetal bovine serum; \\ IncRNA: Long non-coding RNAs; miRNAs: MicroRNAs; mRNA: Messenger RNA; OS: Osteosarcoma; PBS: Phosphate-buffered saline; piRNAs: Piwi-interacting RNAs; RT-PCR: Real-time polymerase chain reaction; siRNAs: Short interfering RNAs; snoRNAs: Small nucleolar RNAs}

\section{Acknowledgements \\ Thanks to Wu Han University. \\ Funding \\ None. \\ Availability of data and materials \\ None.}

\section{Authors' contributions}

The manuscript is an original work of ZF and PH. All data, tables, figures, etc. used in the manuscript are prepared by $\mathrm{ZF}$ and $\mathrm{PH}$, otherwise the sources are cited and reprint permission is attached. ZF and PH read and approved the final manuscript.

\section{Authors' information}

M.D. Zhang Fei, the first author of this paper, is a clinical doctor of The Third Affiliated Hospital of Inner Mongolia Medical University. He is working under the guidance of Peng Hao in the Renmin Hospital of Wuhan University. His work focuses on the basic research on orthopaedics. fjogph@sina.com. M.D. Peng Hao, the correspondence author of this paper, is the chief of clinical research lab in the Orthopaedics Department of Renmin Hospital of Wuhan University. His research focuses on the osteosarcoma.

owujn|@sina.com. 


\section{Competing interests}

The authors declare that they have no competing interests.

\section{Consent for publication}

Permissions from all participants were obtained to publish the data.

\section{Publisher's Note}

Springer Nature remains neutral with regard to jurisdictional claims in published maps and institutional affiliations.

Received: 8 April 2017 Accepted: 17 June 2017

Published online: 05 July 2017

\section{References}

1. Link MP. Osteosarcoma in adolescents and young adults: new developments and controversies. Commentary on the use of presurgical chemotherapy. Cancer Treat Res. 1993;62:383-5.

2. Luetke A, Meyers PA, Lewis I, Juergens H. Osteosarcoma treatment-where do we stand? A state of the art review. Cancer Treat Rev. 2014:40:523-32.

3. Mirabello L, Troisi RJ, Savage SA. Osteosarcoma incidence and survival rates from 1973 to 2004: data from the Surveillance, Epidemiology, and End Results Program. Cancer. 2009;115:1531-43.

4. Farfalli GL, Albergo Jl, Lobos PA, Smith DE, Streitenberger PD, Pallotta Rodriguez MG, Aponte-Tinao LA. Osteosarcoma lung metastases. Survival after chemotherapy and surgery. Medicina (B Aires). 2015;75:87-90.

5. Ferguson WS, Goorin AM. Current treatment of osteosarcoma. Cancer Invest. 2001;19:292-315.

6. Jaffe N. Osteosarcoma: review of the past, impact on the future. The American experience. Cancer Treat Res. 2009;152:239-62.

7. Perkel JM. Visiting "noncodarnia". Biotechniques. 2013:54:301. 303-304.

8. Zhou M, Zhao H, Wang Z, Cheng L, Yang L, Shi H, Yang H, Sun J. Identification and validation of potential prognostic IncRNA biomarkers for predicting survival in patients with multiple myeloma. J Exp Clin Cancer Res. 2015;34:102.

9. Z Zhang Q, Geng PL, Yin P, Wang XL, Jia JP, Yao J. Down-regulation of long non-coding RNA TUG1 inhibits osteosarcoma cell proliferation and promotes apoptosis. Asian Pac J Cancer Prev. 2013;14:2311-5.

10. Kim KH, Roberts CW. Targeting EZH2 in cancer. Nat Med. 2016;22:128-34.

11. Simon JA, Lange CA. Roles of the EZH2 histone methyltransferase in cancer epigenetics. Mutat Res. 2008;647:21-9.

12. Chang CJ, Yang JY, Xia W, Chen CT, Xie X, Chao CH, Woodward WA, Hsu JM, Hortobagyi GN, Hung MC. EZH2 promotes expansion of breast tumor initiating cells through activation of RAF1-beta-catenin signaling. Cancer Cell. 2011;19:86-100.

13. Kleer CG, Cao Q, Varambally S, Shen R, Ota I, Tomlins SA, Ghosh D, Sewalt RG, Otte AP, Hayes DF, et al. EZH2 is a marker of aggressive breast cancer and promotes neoplastic transformation of breast epithelial cells. Proc Natl Acad Sci U S A. 2003:100:11606-11.

14. Varambally S, Dhanasekaran SM, Zhou M, Barrette TR, Kumar-Sinha C, Sanda MG, Ghosh D, Pienta KJ, Sewalt RG, Otte AP, et al. The polycomb group protein EZH2 is involved in progression of prostate cancer. Nature. 2002;419:624-9.

15. Chen YF, Pan KW, Wang PZ, Cao ZY, Wang WB, Wang SY, Hu NG, Xue JH, Li $H$, Jiang $W$, et al. HBP1-mediated regulation of p21 protein through the Mdm2/p53 and TCF4/EZH2 pathways and its impact on cell senescence and tumorigenesis. J Biol Chem. 2016:291:12688-705.

16. Kuroki H, Hayashi H, Okabe H, Hashimoto D, Takamori H, Nakahara O, Nakagawa S, Fukushima Y, Chikamoto A, Beppu T, et al. EZH2 is associated with malignant behavior in pancreatic IPMN via p27(Kip1) downregulation. Plos One. 2014;9(8):e100904.

17. Li K, Chen MK, Situ J, Huang WT, Su ZL, He D, Gao X. Role of co-expression of c-Myc, EZH2 and p27 in prognosis of prostate cancer patients after surgery. Chin Med J. 2013;126:82-7.

18. Kretz M, Webster DE, Flockhart RJ, Lee CS, Zehnder A, Lopez-Pajares V, Qu K, Zheng GX, Chow J, Kim GE, et al. Suppression of progenitor differentiation requires the long noncoding RNA ANCR. Genes Dev. 2012;26:338-43.

19. Merola E, Mattioli E, Minimo C, Zuo WN, Rabitti C, Cicala M, Caviglia R, Pollice L, Gabbrielli A, Giordano A, Claudio PP. Immunohistochemical evaluation of pRb2/p130, VEGF, EZH2, p53, p16, p21(waf-1), p27, and PCNA in Barrett's esophagus. J Cell Physiol. 2006;207:512-9.
20. Mattioli E, Vogiatzi P, Sun A, Abbadessa G, Angeloni G, Ugo DD, Trani D, Gaughan JP, Vecchio FM, Cevenini G, et al. Immunohistochemical analysis of pRb2/p130, VEGF, EZH2, p53, p16(INK4A), p27(KIP1), p21(WAF1), Ki-67 expression patterns in gastric cancer. J Cell Physiol. 2007;210:183-91.

21. Picci P. Osteosarcoma (osteogenic sarcoma). Orphanet J Rare Dis. 2007;2:6.

22. Caudill JS, Arndt CA. Diagnosis and management of bone malignancy in adolescence. Adolesc Med State Art Rev. 2007;18:62-78. IX

23. Hibino S, Saito $Y$, Muramatsu T, Otani A, Kasai $Y$, Kimura M, Saito H. Inhibitors of enhancer of zeste homolog 2 (EZH2) activate tumor-suppressor microRNAs in human cancer cells. Oncogenesis. 2014;3:e104.

\section{Submit your next manuscript to BioMed Central and we will help you at every step:}

- We accept pre-submission inquiries

- Our selector tool helps you to find the most relevant journal

- We provide round the clock customer support

- Convenient online submission

- Thorough peer review

- Inclusion in PubMed and all major indexing services

- Maximum visibility for your research

Submit your manuscript at www.biomedcentral.com/submit
Ciomed Central 